\title{
A WATTHOUR METER METHOD OF TESTING INSTRUMENT TRANSFORMERS
}

\author{
By P. G. AgNew
}

Attempts to determine the constants of instrument transformers by using wattmeters or watthour meters in both the primary and secondary circuits have not met with much success. Modern transformers have such good characteristics that the small differences to be measured are more or less masked by the unavoidable errors of measurement. Moreover, such a method is not applicable to high voltage or large current ranges, which are commercially the more important.

Modern induction watthour meters can, however, be used to determine the difference in ratios, and also the difference in phase angles of either two voltage transformers or of two current transformers, provided the two transformers are of the same range. If, then, the ratio and phase angle of one of the transformers are known, it may be treated as a standard transformer and the constants of other transformers determined in terms of the constants of the standard.

Several modifications of the potentiometer method are available for the precise determination of ratio and phase angle, for the most part making use of laboratory instruments. ${ }^{1}$

\section{OUTLINE OF METHOD}

For voltage transformers an auxiliary current is passed in series through the current coils of the two meters, which are duplicates, and the voltage coil of each meter is connected to one

\footnotetext{
${ }^{1}$ Agnew and Fitch, this Bulletin, 6, p. 281, 1909, Reprint No. 130; Electrical World, 54, p. 1042, 1909. E. Orlich, ETZ, 30, p. 435, 466, I909. L. T. Robinson, Trans. Amer. Inst. Elec. Eing., 28, p. 1005, 1909. F. A. Laws, Elec. World, 55, p. 223, Igro. Sharp and Crawford, Trans. Am. Inst. Elec. Eng., 29, p. 1517, 1910. Agnew and Silsbee, Proc. Am. Inst. Elec. Eng., 31, p. 1267, 1912. Schering and Alberti, Archiv für Elektrotechnik, 2, p. 263, 1914. A method of testing voltage transformers adapted to central-station use and requiring only portable instruments has been described by Brooks, this Bulletin, 10, p. 4I9, I9I4, Reprint No. 217; Electrical World, 62, p. 898, 1913.
} 
of the transformers. If the meters were adjusted to precisely the same rate, the ratios of the transformers would be inversely proportional to the number of rotations of the meters in a given time. Practically the meters can not be adjusted to precisely the same rate, but the difference in rates may be eliminated by interchanging the meters.

The difference in the phase angles may also be obtained by changing the phase of the auxiliary current so that the meters are working on low power factor, since this difference in phase makes the meters run at different speeds as they are connected to one or

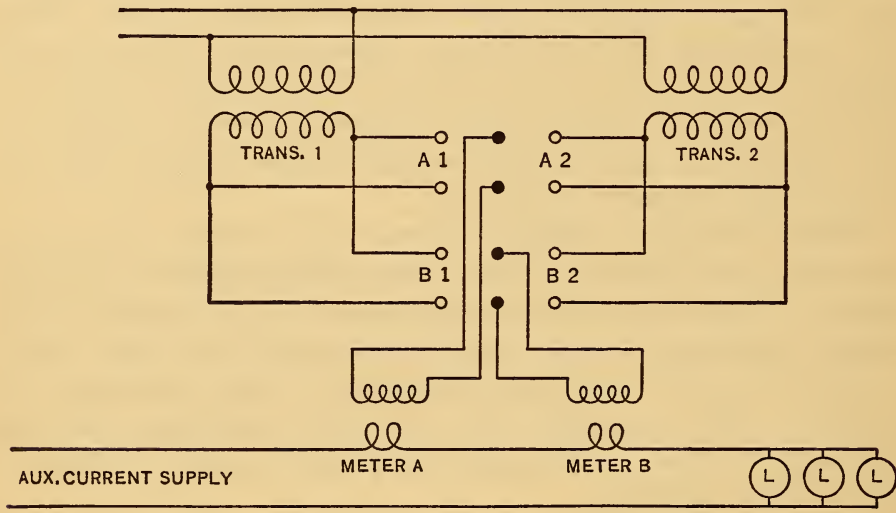

FIG. I.-Arrangement for testing voltage transformers

the other of the transformers. From this data and a knowledge of the difference in ratios obtained at unity power factor the difference in phase angles can be calculated. A diagram of connections for testing a voltage transformer by this method is shown in Fig. I. The ratio measurement may be carried out with a singlephase source of supply. If a three-phase source is available, it is convenient to use a lamp bank for the auxiliary current, putting it on the same phase with the transformers for the ratio measurement and on either of the other phases for the phase angle measurement.

Fig. 2 shows the arrangement of circuits for testing current transformers. The use of the current and the voltage coils of the 
meters is inverted in respect to their use in the case of the voltage transformer. The primaries of the current transformers are in series, and the current coils of the two meters are connected alternately to the two transformers. An auxiliary voltage is applied to the voltage coils of the meters. Otherwise the tests are carried out in the same way as for the voltage transformer.

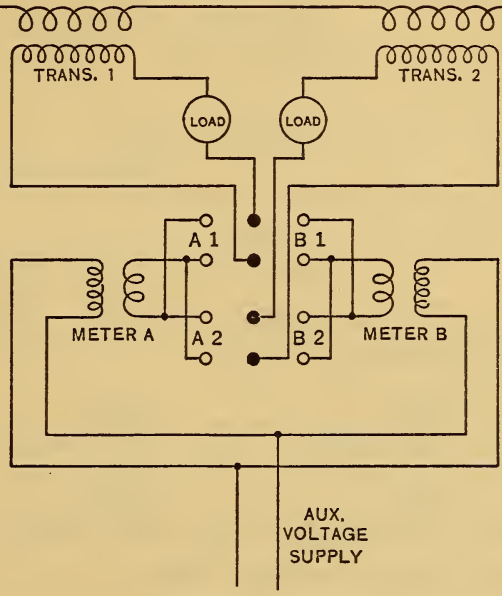

FIG. 2.-Arrangement for testing current transformers

Let $m_{\mathrm{a}}, m_{\mathrm{b}}=$ the rates of the two meters (the rate being the ratio of the recorded watthours to the true watthours). $k=$ the disk constant of the meters (nominal watthours per revolution).

$R_{1}, R_{2}=$ ratios of transformers.

$\alpha_{1}, \alpha_{2}=$ phase angles of transformers (positive for the secondary leading opposition to the primary).

$a_{1}, a_{2}=$ number of turns made by meter $\mathrm{A}$ when connected to transformers $\mathrm{I}$ and 2, respectively.

$b_{1}, b_{2}=$ the same for meter $\mathrm{B}$.

$\cos \theta=$ power factor.

Consider the case of a meter connected to a voltage transformer, and working on low power factor, current lagging. In making $t$ 
turns a meter records $t k$ watt hours. Taking into account only the rate of the meter, $m$, and the ratio of the transformer, $R$, this gives for the energy represented by the primary voltage and the auxiliary current $\frac{t k}{m} R$. But the meter is working at a power factor of $\cos (\theta+\alpha)$ instead of $\cos \theta$, and hence the expression for the energy finally becomes

$$
\frac{t k R \cos \theta}{m \cos (\theta+\alpha)}
$$

or:

$$
\frac{t k R}{m \cos \alpha(\mathrm{I}-\tan \alpha \tan \theta)}
$$

or, since $\alpha$ is very small and hence its cosine may be taken as unity,

$$
\frac{t k R}{m(\mathrm{I}-\tan \alpha \tan \theta)} \text { approximately. }
$$

Applying this to a set of readings taken by interchanging the meters on the transformers

$$
\begin{aligned}
& \frac{a_{1} k R_{1}}{m_{a}\left(\mathrm{I}-\tan \theta \tan \alpha_{1}\right)}=\frac{b_{2} k R_{2}}{m_{b}\left(\mathrm{I}-\tan \theta \tan \alpha_{2}\right)} \\
& \frac{a_{2} k R_{2}}{m_{a}\left(\mathrm{I}-\tan \theta \tan \alpha_{2}\right)}=\frac{b_{1} k R_{1}}{m_{b}\left(\mathrm{I}-\tan \theta \tan \alpha_{1}\right)}
\end{aligned}
$$

from which it may easily be shown ${ }^{2}$ that

$$
\frac{R_{2}}{R_{1}}=\sqrt{\frac{a_{1}}{a_{2}} \cdot \frac{b_{1}}{b_{2}}}
$$

2 From (I) and (2)

$$
\frac{a_{1}}{a_{2}} \cdot \frac{R_{1}}{R_{2}} \cdot \frac{\mathrm{r}-\tan \theta \tan \alpha_{2}}{\mathrm{r}-\tan \theta \tan \alpha_{1}}=\frac{b_{2}}{b_{2}} \cdot \frac{R_{2}}{R_{1}} \cdot \frac{\mathrm{r}-\tan \theta \tan a_{1}}{\mathrm{r}-\tan \theta \tan \alpha_{2}}
$$

which reduces to equation (3) for unity power factor. Since the products of the tangents in (A) are small, it may be written approximately from which (4) immediately follows.

$$
\mathrm{I}+2 \tan \theta\left(\tan a_{1}-\tan \alpha_{2}\right)=\frac{a_{2}}{a_{1}}, \frac{b_{2}}{b_{1}}\left(\frac{R_{2}}{R_{1}}\right)^{2}
$$

(5) may be obtained from (3) by noting that the latter may be written in the form

or

$$
\begin{aligned}
& \frac{R_{2}}{R_{1}}=\sqrt{\left(\mathrm{I}+\frac{a_{1}-a_{2}}{a_{2}}\right)\left(\mathrm{I}+\frac{b_{1}-b_{2}}{b_{2}}\right)} \\
& \quad \frac{R_{2}-R_{1}}{R_{1}}=\frac{1}{2}\left(\frac{a_{1}-a_{2}}{a_{2}}+\frac{b_{1}-b_{2}}{b_{2}}\right) \text { approximately. }
\end{aligned}
$$

which is equation (5).

Similarly (4) becomes

$$
\begin{aligned}
\tan a_{2} & =\tan a_{1}+\frac{I}{2 \tan \theta}\left[I-\left(I+\frac{a_{2}-a_{1}}{a_{1}}\right)\left(I+\frac{b_{2}-b_{1}}{b_{1}}\right)\left(I+\frac{R_{2}-R_{1}}{R_{1}}\right)^{2}\right] \\
& =\tan a_{1}+\frac{I}{\tan \theta}\left[\frac{a_{1}-a_{2}}{2 a_{1}}+\frac{b_{1}-b_{2}}{2 b_{1}}-\frac{R_{2}-R_{1}}{R_{1}}\right] \text { approximately. }
\end{aligned}
$$

Since there are 3438 minutes in a radian (B) reduces to ( 6 ). 
where the $a$ 's and $b$ 's are readings taken at unity power factor, and that

$$
\tan \alpha_{2}-\tan \alpha_{1}=\frac{\mathrm{I}}{2 \tan \theta}\left(\mathrm{I}-\frac{a_{2}}{a_{1}} \cdot \frac{b_{2}}{b_{1}} \cdot \frac{R_{2}{ }^{2}}{R_{1}{ }^{2}}\right)
$$

where the $a$ 's and $b$ 's are now readings taken at low power factor.

(3) is the general formula for the ratio $R_{2}$ in terms of the ratio $R_{1}$, and (4) is the general formula for the phase angle $\alpha_{2}$ in terms of $\alpha_{1}$ and the ratio of the ratios.

These may easily be put into convenient form for slide rule computation by making a few allowable approximations, ${ }^{2}$ giving for the difference in ratios expressed as a fraction of the ratio of the standard transformer

and for the phase angle

$$
\frac{R_{2}-R_{1}}{R_{1}}=\frac{\mathrm{I}}{2}\left[\frac{\left(a_{1}-a_{2}\right)}{a_{2}}+\frac{\left(b_{1}-b_{2}\right)}{b_{2}}\right]
$$

$$
\alpha_{2} \text { (in minutes) }=\alpha_{1}+\frac{3438}{\tan \theta}\left[\frac{a_{1}-a_{2}}{2 a_{1}}+\frac{b_{1}-b_{2}}{2 b_{1}}-\frac{R_{2}-R_{1}}{R_{1}}\right]
$$

Equations (5) and (6) are the working formulas for ratio and phase angle, respectively, and may ordinarily be used instead of the more exact formulas (2) and (3). The approximations involved will amount to less than o.I per cent in ratio for differences in the formula not exceeding 3 per cent, and to less than o.or per cent in ratio for differences not exceeding I per cent.

Equation (6), which gives the phase angle, has its signs correct for voltage transformers connected to meters working on lagging current, and for current transformers connected to meters working on leading current. If the conditions are vice versa, the + sign before the bracketed expression should be changed to - However, it may often be more convenient not to depend upon this relation, but to use the following facts as criteria to experimentally determine whether the transformer under test has a greater or a smaller phase angle than the standard transformer:

I. Adding a noninductive load to a voltage transformer always tends to lag the secondary voltage.

2. Adding noninductive resistance in the secondary of a current transformer tends to advance the phase of the secondary current. 


\section{EXPERIMENTAL RESULTS ${ }^{3}$}

Fig. 3 shows the results of the test of a 5500/ I Io volt transformer by means of two watthour meters and another transformer used as a standard, compared with the results of a precision laboratory method. ${ }^{4}$ The full lines represent the results of the laboratory method and the isolated points those of the watthour meter

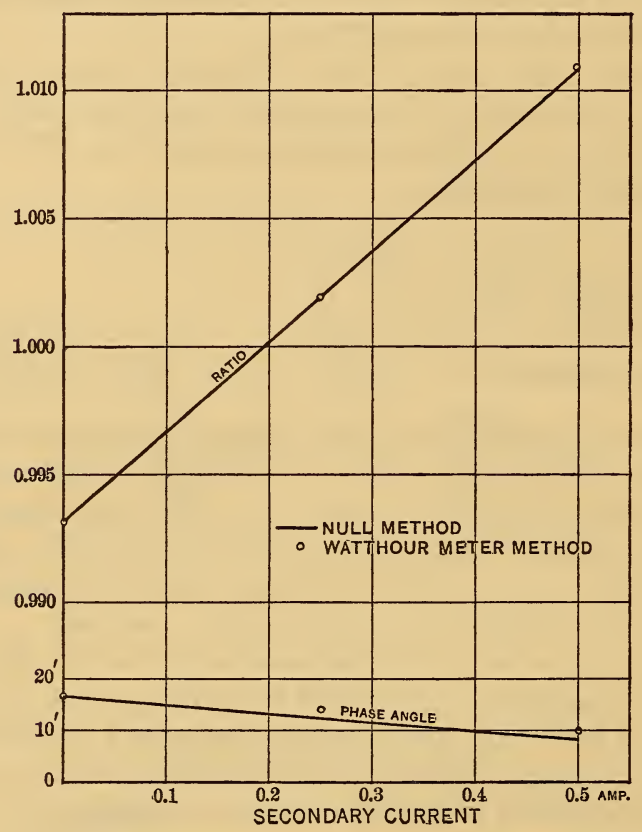

FIG. 3.-Ratio and phase angle of voltage transformercomparison of watthour meter method with null method

method. Each point is the average of two runs of roo turns each, and readings were taken to o.or turn.

Similarly, Fig. 4 shows the results of a comparison of the two methods for a 25 to 5 ampere current transformer. Each point is

3 The author is indebted to W. H. Stannard for assistance in the experimental work.

4 Agnew and Silsbee, Proc. Am. Inst. Elec. Eng., 31, p. 1267, June, 1912. 
here also the average of two runs of roo turns each, excepting at the lower currents where fewer turns were taken.

The meters used were of the type known commercially as $K_{5}$, the disks of which had been graduated in hundredths of a revolution.

It will be seen that the accuracy obtained is greater than is required in commercial power measurements. The method is easily capable of determining ratio to 0.02 or 0.03 per cent, and phase angle to one or two minutes.

It has been found possible to more than double the speed of the meters by shunting the magnets by small pieces of soft iron and

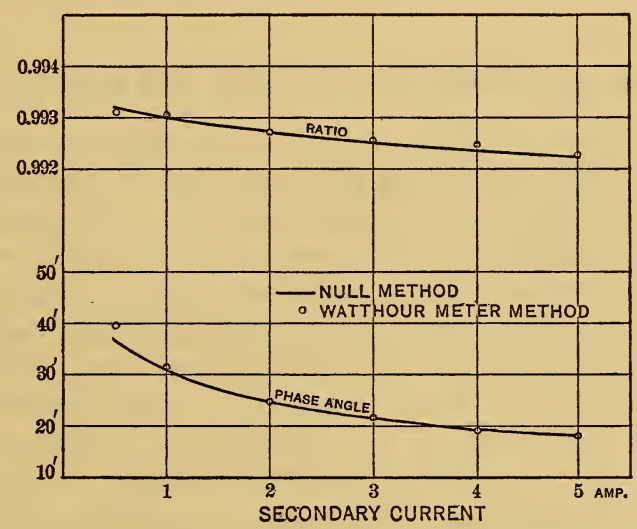

Frg. 4.-Ratio and phase angle of current transformercomparison of watthour meter method with null method

yet get equally accurate results. It is not generally realized that under the very best conditions modern induction meters will repeat consecutive runs to a precision of about o.or per cent at full load. This is clearly shown by the following observations taken with the meters in parallel on the same load. The tenths of a division (thousandths of a revolution) were estimated in taking readings. The speed of the meters had been increased to double normal value by shunting the magnets. 


\begin{tabular}{|c|c|c|c|}
\hline \multirow{2}{*}{ Run } & \multirow{2}{*}{ Per cent of full load } & \multicolumn{2}{|c|}{ Number of turns } \\
\hline & & Meter A & Meter B. \\
\hline $1 \ldots \ldots \ldots \ldots \ldots$ & 100 & 100 & 100.126 \\
\hline $2 \ldots \ldots \ldots \ldots \ldots \ldots \ldots \ldots \ldots \ldots$ & 100 & 100 & .123 \\
\hline 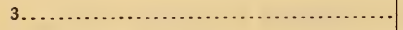 & 100 & 100 & .124 \\
\hline 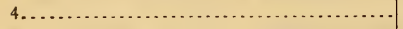 & 100 & 100 & .127 \\
\hline 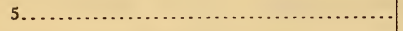 & 100 & 100 & .140 \\
\hline 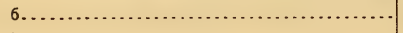 & 100 & 100 & .135 \\
\hline 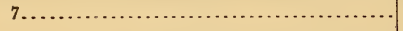 & 100 & 100 & .136 \\
\hline 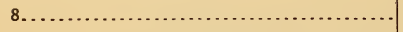 & 10 & 10 & 10.063 \\
\hline 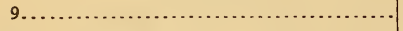 & 10 & 10 & .067 \\
\hline 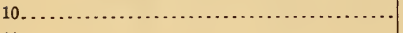 & 10 & 10 & .061 \\
\hline 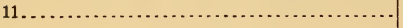 & 10 & 10 & .068 \\
\hline 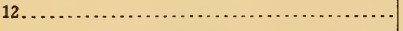 & 10 & 10 & .066 \\
\hline
\end{tabular}

\section{OVER-ALL CORRECTIONS FOR RATIO AND PHASE ANGLE}

There are cases in which it may be convenient to obtain a lump correction for both ratio and phase angle rather than to determine them and to correct for them independently. For example, take the case of a watthour meter and current transformer metering the power supplied to an induction motor. If the standard transformer is inserted in series with the line and readings taken at whatever power factor the system is operating under, then we may consider $R_{2}$ in either of equations 3 or 5 as the combined or over-all power ratio of the transformer. To carry this out in routine work, it would practically be necessary to have a set of curves for the standard transformer giving this ratio calculated from ratio and phase angle for various power factors. ${ }^{5}$ Yet,

${ }^{5} \mathrm{~A}$ discussion of the calculation of the errors introduced by the phase angles of instrument transformers is given by L. T. Robinson, Trans. Am. Inst. Elec. Eng., 28, p. 1005, 1909. Corrections are tabulated for various power factors and phase angles. Similar tables are given in the Meter Code, p. 125, 1912, and in the Meternan's Handbook, p. 296, I9r2.

The matter may be put very briefly in the form

True watt hours $=x+\tan \left(\alpha_{0}-\alpha_{v}\right) \cdot \tan \theta \times$ apparent watthours

where $\alpha_{c}$ and $\alpha_{\nabla}$ are the phase angles of the current and voltage transformers respectively, being positive for the reversed secondary (current or voltage) leading the primary. That the correction factor takes this form, with but a slight approximation may be seen from the reasoning used in the text in deducing equations $\mathrm{r}$ and 2. $\theta$ is to be counted positive for current leading and negative for current lagging. For example, if we have only a voltage transformer whose reversed secondary voltage lags $20^{\prime}$ behind the primary voltage, and if the power factor of the load is 0.5 , current lagging, the correction factor due to phase angle is

$$
\left[I+\tan \left\{0-\left(-20^{\prime}\right)\right\} \tan \left(-60^{\circ}\right)\right]=[x+(+0.0058)(-x .73)]
$$

or $1.0 \%$ is to be subtracted from the reading.

$$
=[\mathrm{r}-0.0100]
$$


since these curves would be computed once for all, for the standard transformer, it would obviate the necessity of going through a somewhat complicated computation for each transformer tested. Of course a similar method could be developed for the case of the voltage transformer, but the complications involved would hardly make it worth while for the voltage transformer alone. In fact, good voltage transformers, at least at 60 cycles, have such small phase angles that in most work the errors introduced by them is no greater than the uncertainty due to other causes.

This process may be extended to the case in which both current and voltage transformers are used. For such a test one meter would be connected to both of the standard transformers (current and voltage), the other to both the transformers under test. Either of equations 3 or 5 will in this case give the over-all power ratio of the two transformers under test $\left(\mathrm{R}_{2}\right)$, for the given condition in terms of the corresponding over-all ratio for the two standard transformers $\left(R_{1}\right)$. This latter quantity could be taken from curves calculated for the two standard transformers, as mentioned above. Experimentally the process is simpler than would appear at first sight since no auxiliary current or voltage is required. This method should prove useful in checking transformers used in the metering of large blocks of power, either single phase or 3 phase.

\section{MISCELLANEOUS DETAIIS}

The use of two of the portable watthour meters so largely used in meter testing is much more convenient than that of house type meters with graduated disks, as the trouble of counting turns is eliminated. However, for economy of time in testing current transformers rated at 5 amperes secondary current, a 5 -ampere watthour meter is preferable to one of a ro-ampere range, as the length of time required for a test with a given accuracy is roughly only half as great. In general, a I or 2 ampere range can not be used on the light loads, as the impedance which would be introduced in the secondary of the transformer would be prohibitive. ${ }^{6}$

\footnotetext{
${ }^{6}$ For the same design a 2 -ampere instrument has as much impedance as six 5 -ampere instruments, and a r-ampere instrument has as much impedance as twenty-five 5 -ampere instruments.
} 
Of course, in the case of a voltage transformer the range is immaterial, as the current is an auxiliary one.

In testing current transformers it is very convenient to use as a source of current a step-down transformer giving but a few volts on the secondary but having ample secondary current capacity. But in such an arrangement if the resistance in the secondary circuit is very low the current may lag very appreciably behind the voltage. This will produce a small error in the ratio measurement, but such a condition is readily detected by the measuring instruments in the circuit. The best way to overcome this difficulty is by the use of a phase-shifting transformer as a source of the auxiliary voltage. Such a transformer is of very great utility for many other purposes, such, for example, as testing meters at low power factor. If such a device is not available, the error may be eliminated by a process of successive approximation. To do this, compute ratio and phase angle from the data just as if the ratio had been determined at unity power factor, and compute the phase angle at whatever power factor the instruments show. Let these be $R_{2}^{\prime}$ and $\alpha_{2}^{\prime}$, and suppose the power factor in the ratio measurement was $\cos \theta_{0}$ instead of unity. By applying equations 5 and $\mathrm{B}$ to this case, using $\theta_{0}$ instead of $\theta$, a little consideration will show that

which may be solved for $R_{2}$,

$$
\tan \alpha_{2}^{\prime}=\tan \alpha_{1}+\frac{\mathrm{I}}{\tan \theta_{0}}\left[\frac{R_{2}^{\prime}-R_{1}}{R_{1}}-\frac{R_{2}-R_{1}}{R_{1}}\right]
$$

$$
R_{2}=R_{2}^{\prime}-R_{1} \tan \theta_{0}\left(\tan \alpha^{\prime}{ }_{2}-\tan \alpha_{1}\right)
$$

With this second approximation to the value of $R_{2}$, we may recompute $\alpha_{2}$.

Care must be used never to open the secondary circuit of a current transformer while current is passing through the primary, for if this is done the properties of the iron will be altered and the ratio and phase angle increased. ${ }^{7}$ In case a transformer is accidentally open circuited it may be brought back to its normal condition by carefully demagnetizing.

It is well to open both current and voltage circuits of the meters at the end of a run, as some meters are more apt to creep on current alone than on voltage alone. 
It is important that the ratio and the phase angle of the standard transformer, whether of the current or voltage type, be determined under actual working conditions of load, including the meter. Multiple-range transformers are very convenient as standards and good transformers have very accurately the same constants for the different series-parallel arrangements of coils. If the no-load ratio of a voltage transformer is required, it may be obtained very closely by adding a second or duplicate meter as load, and then extrapolating to the no-load condition.

While the present method has neither the high precision nor the elegance of the null laboratory methods, it has ample accuracy for commercial requirements, it is independent of ordinary line fluctuations, and no specialized apparatus is required.

WASHINGTON, July I8, I9I4. 